\title{
Self-consistent profile modification in the underdense region of laser-produced plasmas
}

\author{
By J. R. SANMARTÍN AND J. L. MONTAÑES \\ Escuela Técnica Superior de Ingenieros Aeronáuticos, Universidad \\ Politécnica de Madrid, Spain
}

Profile modification in the underdense region of laser-plasmas with spatially uniform temperature $T_{e}$, is studied. A multiple scale method is used to describe self-consistently the plasma flow and the wave field, in (i) the scale of the field wavelength, and (ii) the overall expansion scale. For $T_{e} \propto t^{\alpha}$ ordinary differential equations with definite boundary conditions are obtained. For $\alpha=0$ and weak fields, we explicitly solve the equations and relate the field in the critical layer to the incident field.

\section{Introduction}

In the last few years, considerable attention has been given, both in experimental (Kim, Stenzel \& Wong 1974; Donaldson \& Spalding 1976; Attwood et al. 1978 ) and in theoretical or numerical (Kidder 1972; Forslund et al. 1976; Mulser \& Van Kessel 1977; Max \& MoKee 1977) work, to the question of how radiation pressure affects the expansion flow of laser-produced plasmas. For spatially uniform electron temperature $\left(T_{e}\right)$, as in the case of high enough radiation flux (Sanmartín \& Barrero 1978a,b), and negligible absorption, some definite results are already available: a solution to the wave and plasma flow equations, describing a transition from an oscillatory field to an evanescent one in a thin layer around the critical density $\left(n_{c}\right)$, was given by Lee et al. (1977) in terms of the first maximum of the standing-wave electric field $\left(\widehat{E}_{s c}\right)$ in the underdense side of the layer. In addition, the authors (1980) showed recently that (i) transitions other than that studied by Lee et al. (Mulser \& van Kessel 1977; Max \& MoKee 1977) are not possible, and (ii) the overdense flow, where there is no radiation, adjusts itself for such a universal transition in a manner critically dependent on how $T_{e}$ changes with time; for $T_{e} \propto t^{\alpha}$, both the transition layer and the overdense flow may be considered entirely determined in terms of $\widehat{\mathbb{A}}_{s c}$.

In this paper we use an asymptotic, multiple-scale method to analyze both the fine structure (Faehl \& Kruer 1977) and the overall behaviour of the underdense flow. The analysis shows how to relate (self-consistently) $\widehat{E}_{s c}$ to the incident field in vacuum (ad hoc WKB approximations, used in the literature merely to determine $\widehat{E}_{s c}$, are unjustified because the dielectric function is changing in the scale of the field itself). The general procedure used here, to solve simultaneously the equations for the wave field and the plasma motion, appears to have considerable 
interest; solutions known to the authors for the interaction of an electromagnetic wave and an inhomogeneous dielectric take the dielectric-constant profile as given.

\section{Basic equations}

For no absorption and uniform $T_{e}$, an expanding quasi-neutral plasma interacting with incident light has three conservation equations, which in planar geometry read.

$$
\begin{gathered}
\frac{\partial n}{\partial t}+\frac{\partial}{\partial x}(n v)=0 \\
\frac{\partial}{\partial t}(n v)+\frac{\partial}{\partial x}\left[n v^{2}+n c_{T}^{2}+\frac{\widehat{E}^{2} \cos ^{2} \theta+(c / \omega)^{2}(\partial \widehat{E} / \partial x)^{2}}{16 \pi m}\right]=0 \\
\frac{\partial v}{\partial t}+\frac{\partial}{\partial x}\left(\frac{v^{2}}{2}+c_{T}^{2} \ln n+\frac{\widehat{E}^{2}}{16 \pi m n}\right)=0
\end{gathered}
$$

where $n, v$ and $m$ are density, velocity and (ion) mass; we introduced $c_{T}^{2} \equiv T_{e} / m$. Equations (1) and (2) are the continuity and momentum equations for the ionelectron fluid, neglecting ion pressure and electron inertia. Equation (3) is used instead of the wave equation; (3) is obtained by dividing (2) by $n$ and rearranging the radiation pressure term by means of the wave equation. In the expansion flow the plasma energy equation just yields $\partial c_{T} / \partial x=0 ; c_{T}(t)$ could be obtained from an overall energy balance, but here is considered to be a given, arbitrary function.

The electric field $E$ has been written as $\operatorname{Re}\{\hat{E} \exp [i(\omega / c) y \sin \theta-i \omega t]\}$, where $\theta$ is the angle of incidence in the $(x, y)$ plane (s-polarization); $\widehat{E}$ may be taken real because the dielectric function, $\varepsilon=1-n / n_{c}$, is real. In a previous paper (Sanmartín \& Montañes 1980), (1)-(3) were derived for the case $\theta=0$, but it is trivial to show that the only modification for arbitrary $\theta$ is the factor $\cos ^{2} \theta$ in (2).

Now, let $\tau$ be a characteristic time for the expansion, typically larger than the wave period $2 \pi / \omega$ by a factor of the order of $10^{6}$; then, both a long $\left(c_{T} \tau\right)$ and a short $(c / \omega)$ characteristic length are involved in (1)-(3). In addition, the equations contain two time scales, $\tau$ and $c / \omega c_{T}$ (though they do not contain the very fast oscillations of frequency $\omega$, present in the wave field and in the electron motion) (Stamper 1975; Thomson, Max \& Estabrook 1975); the fast scale, $c / \omega c_{T}$, arises from the fact that the entire short-length, standing-wave pattern drifts with the slow motion of the transition layer (as for light incident on a moving mirror). Thus, we use a multiple scale method (Nayfeh 1973) to carry out an asymptotic expansion in the parameter $c / \omega c_{T} \tau$. Defining

$$
\xi=\frac{\omega \cos \theta}{c}\left[x-x_{c}(t)\right], \quad t^{1}=t, \quad x^{1}=x,
$$

where $x_{c}$ is the position of some definite feature of the transition or critical layer, such as the first maximum of the standing wave, we have

$$
\frac{\partial}{\partial t}=-\dot{x}_{c} \frac{\omega \cos \theta}{c} \frac{\partial}{\partial \xi}+\frac{\partial}{\partial t^{1}}, \quad \frac{\partial}{\partial x}=\frac{\omega \cos \theta}{c} \frac{\partial}{\partial \xi}+\frac{\partial}{\partial x^{1}} \text {. }
$$


We also write $n=n^{0}+n^{1}+\ldots, v=v^{0}+v^{1} \ldots, \widehat{E}=\hat{E}^{0}+\widehat{E}^{1}+\ldots$. For convenience we leave the slow variables in dimensional form $\left(t^{1}, x^{1}\right)$, and do not use explicitly the parameter $c / \omega c_{T} \tau$.

\section{Multiple scale analysis}

3.1. Short (fast) scale structure

The lowest-order equations, from (1)-(3), are

$$
\begin{aligned}
& -\dot{x}_{c} \frac{\partial n^{0}}{\partial \xi}+\frac{\partial}{\partial \xi}\left(n^{0} v^{0}\right)=0 \\
& -\dot{x}_{c} \frac{\partial}{\partial \xi}\left(n^{0} v^{0}\right)+\frac{\partial}{\partial \xi}\left[n^{0}\left(v^{0}\right)^{2}+n^{0} c_{T}^{2}+\frac{\left(\hat{E}^{0}\right)^{2}+\left(\partial \hat{E}^{0} / \partial \xi\right)^{2}}{16 \pi m} \cos ^{2} \theta\right]=0 \\
& -\dot{x}_{c} \frac{\partial^{0}}{\partial \xi}+\frac{\partial}{\partial \xi}\left(\frac{\left(v^{0}\right)^{2}}{2}+c_{T}^{2} \ln n^{0}+\frac{\left(\widehat{E}^{0}\right)^{2}}{16 \pi m n_{c}}\right)=0 .
\end{aligned}
$$

Here $\dot{x}_{\mathrm{s}}$ and $c_{T}$ are functions of $t^{1}$. Introducing

we get

$$
\frac{\dot{x}_{c}-v^{0}}{c_{T}}=\tilde{M}, \quad \frac{n^{0}}{n_{c} \cos ^{2} \theta}=\nu, \quad \frac{\hat{E}^{0}}{\left(4 \pi m n_{c} c_{T}^{2}\right)^{\frac{3}{2}}}=\mathscr{E},
$$

$$
\begin{gathered}
\nu \tilde{M}=\text { const. }=\nu_{s} \tilde{M}_{s}=\nu_{x} \tilde{M}_{1} \\
\nu\left(\tilde{M}^{2}+1\right)+\frac{\mathscr{E}^{2}}{4}+\frac{1}{4}\left(\frac{\partial \mathscr{E}}{\partial \xi}\right)^{2}=\text { const. }=\nu_{s}\left(\tilde{M}_{s}^{2}+1\right)+\frac{\mathscr{E}_{s}^{2}}{4} \\
g(\tilde{M})+\frac{\mathscr{E}^{2}}{2}=\text { const. }=g\left(\tilde{M}_{s}\right)+\frac{\mathscr{E}_{s}^{2}}{2}=g\left(\tilde{M}_{1}\right) .
\end{gathered}
$$

In (8)-(10), constant means independent of $\xi$ but not of $t^{1}, x^{1}$, and

$$
g(\tilde{M}) \equiv \tilde{M}^{2}-\ln \tilde{M}^{2}-1 .
$$

Notice that $\tilde{M}$ is the local Mach number in a frame moving with the $x_{c}$ plane. We used subscripts $s$ and 1 for the maxima and the nodes of $\mathscr{E}$ in the standing wave, respectively; then $\mathscr{E}_{1}=0, \partial \mathscr{E} /\left.\partial \xi\right|_{s}=0$.

From (8)-(10) we have

$$
\begin{aligned}
\frac{\partial \tilde{M}}{\partial \xi}= \pm & \left.\frac{\tilde{M}}{\tilde{M}^{2}-1}\left[g(\tilde{M})_{1}\right)-g(\tilde{M})\right]^{\frac{1}{2}}\left\{g(\tilde{M})-g\left(\tilde{M}_{s}\right)\right. \\
& \left.+2 v_{1} \tilde{M}_{1}\left[\frac{\left(\tilde{M}_{s}-1\right)^{2}}{\tilde{M}_{s}}-\frac{(\tilde{M}-1)^{2}}{\tilde{M}}\right]\right\}^{\frac{1}{2}} \equiv \pm f\left(\tilde{M}, \tilde{M}_{s}, \tilde{M}_{1}, v_{1}\right) .
\end{aligned}
$$

If $\widetilde{M}$ exceeds unity throughout the underdense region (see discussion later), we have $\widetilde{M}_{1}>\tilde{M}_{s}$, and therefore $\tilde{M}$ oscillates between $\widetilde{M}_{s}$ and $\widetilde{M}_{1}$, with finite period

$$
\Delta \xi=2 \int_{\tilde{M}_{s}}^{\tilde{M}_{1}} f^{-1} d \tilde{M} .
$$


Both $\nu$ and $\mathscr{E}$ are also periodic, with periods $\Delta \xi$ and $2 \Delta \xi$ respectively. Clearly, then, for given $\widetilde{M}_{s}, \widetilde{M}_{1}$ and $\nu_{1}$, system (8)-(10) yields the entire short scale structure. Thus, to complete the solution, we just need to determine $\widetilde{M}_{s}, \widetilde{M}_{1}$ and $\nu_{1}$ as functions of $t^{1}, x^{1}$.

\subsection{Long (slow) scale structure}

Now, the next-order equation from ( 1 ) is

$$
\frac{\partial n^{0}}{\partial t^{1}}+\frac{\partial}{\partial x^{1}}\left(n^{0} v^{0}\right)+\frac{\partial}{\partial \xi}\left[\frac{\omega \cos \theta}{c}\left(n^{0} v^{1}+n^{1} v^{0}-\dot{x}_{c} n^{1}\right)\right]=0 .
$$

Integrating (12) from $\xi_{a}$ to $\xi_{b}$, where $\xi_{b}-\xi_{a}=K \Delta \xi$ and $K$ is an integer, we have

$$
\left.\frac{\omega \cos \theta}{c}\left(n^{1} v^{0}+n^{0} v^{1}-\dot{x}_{c} n^{1}\right)\right|_{\xi_{c}} ^{\xi_{b}}=K \Delta \xi\left\langle\frac{\partial n^{0}}{\partial t^{1}}+\frac{\partial}{\partial x^{1}}\left(n^{0} v^{0}\right)\right\rangle,
$$

where $\left\langle>\right.$ means average over a $\xi$-period; in the preceding integration $x^{1}$ and $t^{1}$ are parameters. If $K \rightarrow \infty$, the asymptotic expansion will break down (either $n^{1} / n^{0}$ or $v^{1} / v^{0}$ will be unbounded), unless the mean value in (13) vanishes; thus, to avoid secularities, we set

$$
\frac{\partial\langle n\rangle}{\partial t}+\frac{\partial}{\partial x}\langle n v\rangle=0
$$

Similar arguments on (2) and (3) yield

$$
\begin{gathered}
\frac{\partial}{\partial t}\langle n v\rangle+\frac{\partial}{\partial x}\left[\left\langle n v^{2}\right\rangle+c_{T}^{2}\langle n\rangle+\frac{\left\langle\hat{E}^{2}\right\rangle+\left\langle(\partial \widehat{E} / \partial \xi)^{2}\right\rangle}{16 \pi m} \cos ^{2} \theta\right]=0, \\
\frac{\partial\langle v\rangle}{\partial t}+\frac{\partial}{\partial x}\left[\frac{\left\langle v^{2}\right\rangle}{2}+c_{T}^{2}\langle\ln \dot{n}\rangle+\frac{\left\langle\widehat{E}^{2}\right\rangle}{16 \pi m n_{c}}\right]=0 .
\end{gathered}
$$

Equations (14)-(16) describe the lowest-order solution in the long, slow scale; we suppressed superseripts for simplicity. The averages may be written in terms of $\tilde{M}_{s}, \tilde{M}_{1}$ and $\nu_{1}$, for given $x_{c}(t)$ and $c_{T}(t)$, by using (7)-(11).

\section{Power-law temperature}

While (14)-(16) may be used under broad conditions, here we shall limit our analysis to the case where the ratio $\widehat{E}_{s c}^{2} / T_{e}$ is constant in time (Virmont, Pellat \& Mora 1978), and $T_{e} \propto t^{\alpha}$; then (14)-(16) have a self-similar solution. Introducing $\eta=x / \int_{0}^{t} c_{T}\left(t^{\prime}\right) d t^{\prime}$, we get $\dot{x}_{\mathrm{c}}=\eta_{\mathrm{c}} c_{T} ; \eta_{\mathrm{c}}$ may be determined from an analysis of the overdense flow, and is a function of $\alpha$ and $\mathscr{E}_{s c} \equiv \mathscr{E}_{s}\left(x_{c}\right)$ which will be taken as known (Sanmartín \& Montañes 1980). Then (14)-(16) become

$$
\begin{gathered}
\tilde{\eta} \frac{d}{d \tilde{\eta}}\left(\nu_{1} \tilde{M}_{1}\langle\tilde{M}-1\rangle\right)+\frac{d}{d \tilde{\eta}} \nu_{1} \tilde{M}_{1}=0, \\
\frac{\alpha}{\alpha+2}\left(\eta_{c}-\langle\tilde{M}\rangle\right)+\tilde{\eta} \frac{d}{d \tilde{\eta}}\langle\tilde{M}\rangle+\frac{d}{d \tilde{\eta}}\left(\frac{\tilde{M}_{1}^{2}}{2}+\ln \nu_{1}\right)=0,
\end{gathered}
$$




$$
\begin{aligned}
\frac{\alpha}{\alpha+2} \nu_{1} \tilde{M}_{1}\left(\eta_{c}\left\langle\tilde{M}^{-1}\right\rangle-1\right) & +\tilde{\eta} \frac{d}{d \tilde{\eta}}\left(\nu_{1} \tilde{M}_{1}\right) \\
+ & +\frac{d}{d \tilde{\eta}}\left[\nu_{1} \tilde{M}_{1} \frac{\tilde{M}_{s}^{2}+1}{\tilde{M}_{s}}+\frac{g\left(\tilde{M}_{1}\right)-g\left(\tilde{M}_{s}\right)}{2}\right]=0
\end{aligned}
$$

where we defined $\tilde{\eta} \equiv \eta-\eta_{c}$. For any function $F(\tilde{M})$ (such as $\tilde{M}$ and $\tilde{M}^{-1}$ ) the mean value is

$$
\langle F(\tilde{M})\rangle=\frac{\int_{\tilde{M}_{s}}^{\widetilde{M}_{1}} F(\tilde{M}) d \tilde{M} / f\left(\tilde{M}, \tilde{M}_{s}, \tilde{M}_{1}, \nu_{1}\right)}{\int_{\widetilde{M}_{s}}^{\tilde{\widetilde{M}} 1} d \tilde{M} / f\left(\tilde{M}, \tilde{M}_{s}, \tilde{M}_{1}, \nu_{1}\right)} .
$$

The boundary conditions for system (17)-(19) follow from the analysis of the critical (transition) layer (Lee el al. 1977; Sanmartín \& Montañes 1980): at $\tilde{\eta}=0\left(x=x_{c}\right)$

and

$$
\tilde{M}_{s}=1, \quad g\left(\tilde{M}_{1}\right)=\mathscr{E}_{s c}^{2} / 2 \quad\left(\tilde{M}_{1}>1\right)
$$

where

$$
\nu_{1}=\nu_{2} \tilde{M}_{2} / \widetilde{M}_{1}
$$

$$
\nu_{2}=\frac{g\left(\tilde{M}_{2}\right)}{2\left(1-\tilde{M}_{2}\right)^{2}}, \quad g\left(\tilde{M}_{2}\right)=\frac{\mathscr{E}_{s c}^{2}}{2} \quad\left(\tilde{M}_{2}<1\right)
$$

Subscript 2 refers to conditions on the evanescent side of the critical layer. Thus, $\eta_{c}, \tilde{M}_{s}(0), \widetilde{M}_{1}(0)$ and $\nu_{1}(0)$ are known, and the system $(17)-(19)$ may be solved, for each pair of values $\alpha$ and $\mathscr{E}_{s c}$. Notice that (8)-(11) with $\tilde{M}_{s}=1$, together with (22), recover the known critical-layer structure and jump conditions.

Concerning that system, the following points should be noticed now. First, $\widetilde{M} \geqslant 1$ at $\tilde{\eta}=0$, and therefore $\tilde{M} \geqslant 1$ everywhere, because if $\tilde{M}$ could get below unity at some $\tilde{\eta}$, then both $\langle\tilde{M}\rangle$ and $\left\langle\tilde{M}^{-1}\right\rangle$ would present discontinuities there. Secondly, if $\nu_{s}$ (and $a$ fortiori $\nu_{1}$ ) decreases to zero as $\tilde{\eta}$ decreases, as expected, then (10) and (11) lead to $\mathscr{E}=\mathscr{E}_{s} \cos \xi$, the behaviour of the field in vacuum. Thirdly, (19) shows that $g\left(\tilde{M}_{1}\right)-g\left(\tilde{M}_{s}\right) \rightarrow$ const. $=\frac{1}{2}\left[\mathscr{E}_{s}(\text { vacuum })\right]^{2}$ as $\nu_{s} \rightarrow 0$; since (17) cannot be satisfied if $\widetilde{M}_{s} \rightarrow$ const. in that limit, both $\tilde{M}_{s}$ and $\widetilde{M}_{1}$ must grow indefinitely as $\nu_{1}$ vanishes. Hence, $\tilde{M}_{1}-\tilde{M}_{s}$ must vanish in such a manner that

$$
\lim _{\nu_{1} \rightarrow 0} \tilde{M}_{1}\left(\tilde{M}_{1}-\tilde{M}_{s}\right)=\text { const. }=\frac{1}{4}\left[\mathscr{E}_{s}(\text { vacuum })\right]^{2}
$$

This equation, together with

$$
2 E_{0}=\widehat{E}_{s}(\text { vacuum })=\left(4 \pi n_{c} T_{e}\right)^{\frac{1}{2} \mathscr{E}_{s}(\text { vacuum }),}
$$

will yield the incident field $E_{0}$. Finally, as $\tilde{M}_{1}-\tilde{M}_{s} \rightarrow 0$, the oscillations in both $\tilde{M}$ and $\nu$ vanish: $\widetilde{M} \simeq \widetilde{M}_{1} \simeq \widetilde{M}_{s}$ and $\nu \simeq \nu_{1} \simeq \nu_{s}$. Defining $u=v / c_{T} \equiv-\tilde{M}+\eta_{c}$, (17) and (18) become

$$
\frac{d}{d \eta} \ln \nu=\frac{1}{\eta-u} \frac{d u}{d \eta}, \quad\left[(\eta-u)^{2}-1\right] \frac{d u}{d \eta}=\frac{\alpha u}{2+\alpha}(\eta-u),
$$

which are just those describing the flow in the absence of radiation (Sanmartín \& Montañes 1980). 


\section{Constant temperature, weak field limit}

For $\alpha=0,(17)-(19)$ form a linear, homogeneous system for $d \tilde{M}_{1} / d \tilde{\eta}, d \tilde{M}_{s} / d \tilde{\eta}$ and $d \nu_{1} / d \tilde{\eta}$. The compatibility condition for the system is a cubic equation for $\tilde{\eta}$ in terms of $\tilde{M}_{s}, \widetilde{M}_{1}$ and $\nu_{1}$ the root that vanishes with $\tilde{M}_{s}-1$ should be introduced in the system to yield two differential equations relating $\widetilde{M}_{s}, \widetilde{M}_{1}$ and $\nu_{1}$.

\subsection{Weak field: the outer region}

Particularly simple is the case $\alpha=0, \mathscr{E}_{s c}$ small. Then, the boundary conditions are

$$
\tilde{M}_{s}(0)=1, \quad \tilde{M}_{1}(0)=1+\frac{1}{2} \mathscr{E}_{s c}, \quad \nu_{1}(0)=1-\frac{5}{6} \mathscr{E}_{s c} .
$$

We expect $\tilde{M}_{1}-\tilde{M}_{s}$ to be small everywhere. Expanding $F(\tilde{M})$ in powers of $\tilde{M}-\tilde{M}_{1}$, we find

$$
\langle F(\tilde{M})\rangle=F\left(\tilde{M}_{1}\right)-\frac{1}{2} F^{\prime \prime}\left(\tilde{M}_{1}\right)\left(\tilde{M}_{1}-\tilde{M}_{s}\right)+\ldots
$$

Expanding the solution to (17)-(19) in powers of $\widetilde{M}_{1}-\widetilde{M}_{s}$, we get to lowest order

$$
\tilde{M}_{1}=\tilde{M}_{s}=1-\tilde{\eta}, \quad \nu_{1}=C e^{\tilde{\eta}}
$$

the boundary conditions require, to this order, $C=1$. This is simply the solution in the absence of radiation (the well-known isothermal expansion). The nextorder corrections lead to

$$
\begin{gathered}
\tilde{M}_{1, s}=1-\tilde{\eta}+\frac{\Delta_{1}}{4\left(1-e^{\tilde{\eta}}\right)^{\frac{1}{2}}}\left[\frac{1}{2} \frac{e^{\tilde{\eta}}}{1-e^{\tilde{\eta}}} \pm \frac{2(1-\tilde{\eta})}{\tilde{\eta}^{2}-2 \tilde{\eta}}\right], \\
\nu_{1}=e^{\tilde{\eta}}+\Delta_{2} e^{\tilde{\eta}}-\frac{\Delta_{1} e^{\tilde{\eta}}}{4\left(1-e^{\tilde{\eta}}\right)^{\frac{3}{2}}}\left[\frac{1}{2} \frac{e^{\tilde{\eta}}}{1-e^{\tilde{\eta}}}+\frac{2-2 \tilde{\eta}+\tilde{\eta}^{2}}{\tilde{\eta}^{2}-2 \tilde{\eta}}\right],
\end{gathered}
$$

where $\Delta_{1}$ and $\Delta_{2}$ are small (unknown) constants that vanish with $\mathscr{E}_{s c}$; in (26) the upper (lower) sign corresponds to the $1(s)$ subscript. Clearly, the boundary conditions cannot be satisfied to order $\mathscr{E}_{s c}$ : an inner, thin region adjoining the origin $\tilde{\eta}=0$ must exist, and the solution there should be matched to (26) and (27) for the outer region according to the method of inner and outer expansions (Nayfeh 1973). The approximation that fails for $\tilde{\eta} \rightarrow 0$ is (25); the failure is due to the fact that, near $\tilde{\eta}=0$, all three $\tilde{M}_{s}, \tilde{M}_{1}$ and $\nu_{1}$ are close to unity and the computation of $\langle F(\tilde{M})\rangle$ becomes more complex.

\subsection{Weak field: the inner region}

We now define inner variables

$$
\begin{gathered}
\zeta=\eta / \mathscr{E}_{s c}, \quad \mu=(\tilde{M}-1) / \mathscr{E}_{s c}, \quad \mu_{s}=\left(\widetilde{M}_{s}-1\right) / \mathscr{E}_{s c}, \\
\mu_{1}=\left(\tilde{M}_{1}-1\right) / \mathscr{E}_{s c}, \quad N=\left(\nu_{1}-1\right) / \mathscr{E}_{s c} .
\end{gathered}
$$

To lowest order, (17)-(19) (with $\alpha=0$ ) become

$$
\frac{d}{d \zeta}\left(N+\mu_{1}\right)=0
$$




$$
\begin{gathered}
\mu_{1} \frac{d \mu_{1}}{d \zeta}+\zeta \frac{d\langle\mu\rangle}{d \zeta}=0 \\
\frac{4}{3} \mu_{1} \frac{d \mu_{1}}{d \zeta}-4 \mu_{s}\left(\frac{1}{3}+\mu_{s}\right) \frac{d \mu_{s}}{d \zeta}-2 \zeta \frac{d\left\langle\mu^{2}\right\rangle}{d \zeta}=0,
\end{gathered}
$$

the boundary conditions being

$$
r_{s}=0, \quad \mu_{1}=\frac{1}{2}, \quad N=-\frac{5}{6} .
$$

Equation (28) yields immediately $N+\mu_{1}=-\frac{1}{3}$. Then the mean value (20) for a function $F(\mu)$ takes the form

where

$$
\langle F(\mu)\rangle=\int_{\mu_{s}}^{\mu_{1}} \frac{F(\mu) d \mu}{\phi} / \int_{\mu_{s}}^{\mu_{1}} \frac{d \mu}{\phi},
$$

$$
\phi\left(\mu, \mu_{s}, \mu_{1}\right)=\mu\left[\left(\mu_{1}^{2}-\mu^{2}\right)\left(\mu-\mu_{s}\right)\left(\mu^{2}+\mu \mu_{s}+\mu_{s}^{2}+\mu+\mu_{s}\right)\right]^{-\frac{1}{2}} .
$$

The compatibility condition for (29) and (30) is a quadratic equation for $\zeta$ in terms of $\mu_{s}$ and $\mu_{1}$. The root that vanishes with $\mu_{s}$ must be introduced in (29), yielding an equation of the form

$$
\frac{d \mu_{s}}{d \mu_{1}}=H\left(\mu_{1}, \mu_{s}\right), \quad \mu_{s}\left(\mu_{1}=\frac{1}{2}\right)=0 .
$$

$H$ is such that $\mu_{1}-\mu_{\mathrm{s}} \rightarrow B / \mu_{1}^{\frac{3}{2}}$, and $\zeta \rightarrow-\mu_{1}$, as $\mu_{1} \rightarrow \infty$; we numerically find $B \simeq 0 \cdot 103$.

\subsection{Weak field: determination of $\mathscr{E}_{s c}$}

Matching the inner and outer solutions allows us to determine $\Delta_{1}$ in (26). We write, for the inner layer,

$$
\left(\tilde{M}_{1}-1\right)^{\frac{3}{2}}\left(\tilde{M}_{1}-\widetilde{M}_{s}\right) \rightarrow B \mathscr{E}_{s c}^{\frac{h}{c}} \quad \text { as } \quad \tilde{\eta} / \mathscr{E}_{s c} \rightarrow-\infty .
$$

If $\Delta_{\mathbf{1}} \sim \mathscr{E}_{s c}^{\frac{5}{5}}$, equation (26), for $1 \gg|\tilde{\eta}| \gg \mathscr{E}_{s c}$, yields

$$
\tilde{M}_{1} \simeq 1-\tilde{\eta}, \quad \tilde{M}_{1}-\tilde{M}_{s} \simeq \Delta_{1} / 2(-\tilde{\eta})^{\frac{3}{2}},
$$

and the matching requires

$$
(-\tilde{\eta})^{\frac{3}{2}} \frac{\Delta_{1}}{2(-\tilde{\eta})^{\frac{3}{2}}} \simeq B \mathscr{E}_{s c}^{\mathscr{E} \frac{5}{2}}, \quad \text { or } \quad \Delta_{1}=2 B \mathscr{E}_{\mathrm{sc}}^{\frac{5}{2}} .
$$

Now, for $\tilde{\eta} \rightarrow-\infty$, (26) leads to

$$
\tilde{M}_{1} \sim-\tilde{\eta}, \quad \tilde{M}_{1}-\tilde{M}_{s} \sim-\Delta_{1} / \tilde{\eta},
$$

so that, from (23), we finally obtain

$$
\frac{1}{4}\left[\mathscr{E}_{s}(\text { vacuum })\right]^{2}=2 B \mathscr{E}_{s c}^{\frac{5}{2}},
$$

which in dimensional form reads

$$
\widehat{E}_{s c} \simeq 1 \cdot 88\left[\left(4 \pi n_{c} T_{e}\right)^{\frac{1}{2}}\right]^{\frac{1}{3}} E_{0}^{\frac{4}{4}} \quad\left(E_{0}^{2} \ll 4 \pi n_{c} T_{e}\right) .
$$




\section{Discussion}

To take into account the radiation pressure in the underdense flow of a laserproduced plasma, we used a multiple-scale method to derive equations describing self-consistently the flow and the wave field in both the overall expansion and the wavelength scales; the procedure given may have a broad validity for the propagation of an electromagnetic wave in a fluid dielectric. Quasi-neutrality, negligible absorption and spatially uniform temperature were assumed. The known structure of the critical layer provided the appropriate boundary conditions. For time power-law temperatures, for which the overdense flow has been analyzed elsewhere, a detailed study was made. For constant temperature and weak field we obtained a maximum flux about $10 \%$ less than that resulting from an ad hoc WKB approximation (Lee et al. 1977).

We are trying now to extend our results to include spherical, $p$-polarization (resonant absorption), and complex dielectric function, effects. Important questions that should also be considered in future work are the stability of the present solution (Valeo \& Estabrook 1975) and its modification in the presence of two electron temperatures.

This work was supported by the Junta de Energía Nuclear of Spain.

\section{REFERENCES}

Atrwood, D. T., Swemney, D. W., AurraACH, J. M. \& LeE, P. H. Y. 1978 Phys. Rev. Lett. 40, 184.

Donaldson, T. P. \& Spalding, I. J. 1976 Phys. Rev. Lett. 36, 467.

FAEHL, R. J. \& KRUER, W. L. 1977 Phys. Fluids, 20, 55.

ForsuUnd, D. W., KrndeL, J. M., Lew, K. \& Lindmax, E. L. 1976 Phys, Rev. Lett. 36, 35. KmDER, R. E. 1972 Lawrence Livermore Laboratory Report UCRL-74040.

Kim, H. C., Stenzet, R. L. \& Wong, A. Y. 1974 Phys. Rev. Lett. 33, 886.

LeE, K., Forslond, D. W., Kindex, J. M. \& Lindman, E. L. 1977 Phys. Fluids, 20, 51.

Max, C. E. \& McKeE, C. F. 1977 Phys. Rev. Lett. 39, 1336.

MULSER, P. \& van KESSEL, C. 1977 Phys. Rev. Lett. 38, 902.

NAXHEH, A. H. 1973 Perturbation Methods, chs. 6 and 4. Wiley.

Sammartín, J. R. \& Barrero, A. 1978 a Phys. Fluids, 21, 1957.

Sammartín, J. R. \& Barrero, A. 1978 b Phys. Fluids, 21, 1967.

Sanmartín, J. R. \& Montañes, J. L. 1980 Phys. Fluids (to be published).

STAMPER, J. A. 1975 Phys. Fluids, 18, 735.

Тномpson, J. J., Max, C. E. \& Estabrook, K. 1975 Phys. Rev. Lett. 35, 663.

Vateo, E. J. \& Estabrook, K. G. 1975 Phys. Rev. Lett. 34, 1008.

Virmont, J., Peliat, R. \& Mora, A. 1978 Phys. Fluids, 21, 567. 